\title{
BMJ Open Assessing the feasibility of a rapid, high-volume cervical cancer screening programme using HPV self-sampling and digital colposcopy in rural regions of Yunnan, China
}

Andrew Goldstein (D) , ${ }^{1,2}$ Lena Sophia Goldstein, ${ }^{3}$ Roberta Lipson, ${ }^{4}$ Sarah Bedell, ${ }^{2}$ Jue Wang, ${ }^{4}$ Sarah A Stamper, ${ }^{5}$ Gal Brenner, ${ }^{6}$ Gail R Goldstein, ${ }^{7}$ Karen Davis O'Keefe, ${ }^{8} \mathrm{~S}$ Casey O'Keefe, ${ }^{9}$ McKenna O'Keefe, ${ }^{10}$ Tierney O'Keefe, $^{8}$ Amelia R Goldstein, ${ }^{11}$ Anna Zhao ${ }^{4}$

To cite: Goldstein A, Goldstein LS, Lipson R, et al. Assessing the feasibility of a rapid, high-volume cervical cancer screening programme using HPV self-sampling and digital colposcopy in rural regions of Yunnan, China. BMJ Open 2020;10:e035153. doi:10.1136/ bmjopen-2019-035153

- Prepublication history for this paper is available online. To view these files, please visit the journal online (http://dx.doi. org/10.1136/bmjopen-2019035153).

Received 22 0ctober 2019 Accepted 19 February 2020
Check for updates

(C) Author(s) (or their employer(s)) 2020. Re-use permitted under CC BY-NC. No commercial re-use. See rights and permissions. Published by BMJ.

For numbered affiliations see end of article.

Correspondence to Dr Andrew Goldstein; obstetrics@yahoo.com

\section{ABSTRACT}

Objective Implementation of a novel, rapid, highvolume, see-and-treat cervical cancer screening programme using self-swab human papillomavirus (HPV) testing and digital colposcopy in underserved regions of Yunnan China.

Design 480-980 women per day self-swabbed for high-risk HPV (hrHPV+). Four careHPV machines (Qiagen) were run simultaneously to test the specimens. All hrHPV+ patients were contacted the same day and digital colposcopy was performed with the enhanced visual assessment system (Mobile0DT). Digital images were obtained, and all suspected lesions were biopsied and then treated.

Setting Rural and underserved areas of the Yunnan province, Kunming municipality.

Participants 3600 women, mean age 50.2 years, who had never been screened for cervical cancer. The women were of the Yi, Hui, Dai and Han ethnicities.

Interventions Cryotherapy was performed on all lesions suspicious for cervical intraepithelial neoplasia (CIN) 1 and loop electrosurgical excision procedure was performed on all lesions suspicious for $\geq$ CIN2. Endocervical curettage was performed if the transformation zone was not fully visualised.

Results 216 women (6\%) were hrHPV+. 168 underwent same-day colposcopy (23 CIN1, 17 $\geq \mathrm{CIN2}$ ). Digital colposcopy was able to identify 15 of $16(93.8 \%) \geq$ CIN2 lesions.

Conclusions This study illustrates a high-volume, rapid and practical strategy that can be used to screen and treat an ethnically diverse group of Chinese women. First, HPV self-sampling allows large numbers of women to be screened rapidly and relatively inexpensively. Only $\mathrm{hrHPV}+$ women will then require further evaluation. Digital colposcopy is then performed on hrHPV+ women with a portable digital colposcope. The high-resolution images obtained can facilitate appropriate same-day treatment as they are able to accurately distinguish between CIN1 and $\geq$ CIN2 lesions.

\section{Strengths and limitations of this study}

- In this high-volume cervical cancer screening study 480-980 women per day self-swabbed for human papillomavirus (HPV).

- Swabs were tested for high-risk HPV (hrHPV) with the careHPV testing system.

- The careHPV system, which relies on signal amplification technology, may be less sensitive than PCRbased systems to detect $\geq$ cervical intraepithelial neoplasia (CIN) 2 lesions on self-swabs.

- Digital colposcopy, using a new, handheld digital colposcope with advanced optics, was performed on hrHPV+ women.

- Cryotherapy was performed on women with lesions suspicious for CIN1 and loop electrosurgical excision procedure was performed on women with lesions suspicious for $\geq$ CIN2.

\section{INTRODUCTION}

Cervical cancer is the leading cause of cancer deaths in women of the developing world. ${ }^{1}$ The development of screening and treatment modalities, as well as primary prevention mechanisms, have dramatically reduced the incidence of and mortality due to cervical cancer in developed nations. Accordingly, up to $85 \%$ of the disease burden thus lies in developing nations. ${ }^{2}$ Currently, there are no national cervical cancer screening programmes in China and only 10\%-30\% of Chinese women report having ever had cervical cancer screening. ${ }^{3}$ As a result, China carries both a high incidence of cervical cancer, of approximately 15.3/100 000, and a mortality rate of $4.57 / 100000 .{ }^{4}$ Recent estimates suggest that the number of new cervical 
cancer cases in China in 2014 was over 102 000, with over 30400 deaths.

Human papillomavirus (HPV) is the most common sexually transmitted infection worldwide, with a prevalence rate of $11 \%-25 \%$, and accounts for virtually all cases of cervical dysplasia and cancer. ${ }^{56}$ The prevalence of HPV varies among different ethnic and geographical regions. In China, the prevalence and genotype distributions of HPV are well documented. HPV genotypes 16, 18, 52, 58 and 59 are the most common strains among Chinese women, with differing distribution rates throughout the country. ${ }^{7-9}$ In general, women living in rural areas are less likely than urban-dwelling women to report ever having had a Pap test, ${ }^{3}$ and mortality rates from cervical cancer are up to $48 \%$ higher in rural areas. ${ }^{10}$

Yunnan Province is a geographically and ethnically diverse region in south-west China, with a population of over 42 million. ${ }^{11}$ Yunnan is a relatively underdeveloped province, and at the time of our visit, contained several poverty-stricken counties. ${ }^{12}$ It is estimated that the prevalence of high-risk HPV (hrHPV) within the Yunnan Province ranges from $7.1 \%$ to $27.4 \%$ which is highly dependent on the ethnicity of the women tested. ${ }^{13}$

Due to the level of development, complex geography and dispersed population across the rural parts of majority of Yunnan Province, the conventional, multistep screening process for cervical cancer is not feasible. The traditional process of screening with cytology (pap smear), colposcopy, biopsy and subsequent treatment of women diagnosed with cervical dysplasia is too resource-intensive for low-income regions. Accordingly, less burdensome techniques such as HPV testing have been studied as screening techniques in low-resource settings. ${ }^{145}$

Digital colposcopes are highly portable and allow for the capture of high-resolution digital images. These images can allow for magnified visualisation of surface morphology of the cervix, especially suspicious areas. Studies have demonstrated its accuracy and ease of use in resource-poor settings. ${ }^{16-19}$ The enhanced visual assessment (EVA) (MobileODT, Israel) system is one example of a digital colposcope that uses the advanced optics found in Android smartphones that are quite common, even in low-resource countries (figure 1).

Cervicovaginal HPV DNA testing is a reliable test for the detection of high-grade cervical intraepithelial neoplasia $(\mathrm{CIN}) .{ }^{14}$ While typically used in conjunction with a Pap smear for traditional cervical cancer screening, the accuracy and high sensitivity for the detection of cervical dysplasia of HPV DNA testing have led to various studies investigating its use as a stand-alone screening strategy in low-resource settings. ${ }^{14}$ Studies have demonstrated that healthcare provider-collected cervical HPV samples are significantly more sensitive and carry only a slightly lower specificity for the detection of high-grade CIN than conventional cytology. ${ }^{20}$ Additionally, HPV DNA test swabs can be obtained by the patient alone ('self-sampling' or 'self-swab'), thereby eliminating the need for a skilled provider and equipment to perform a pelvic exam. This

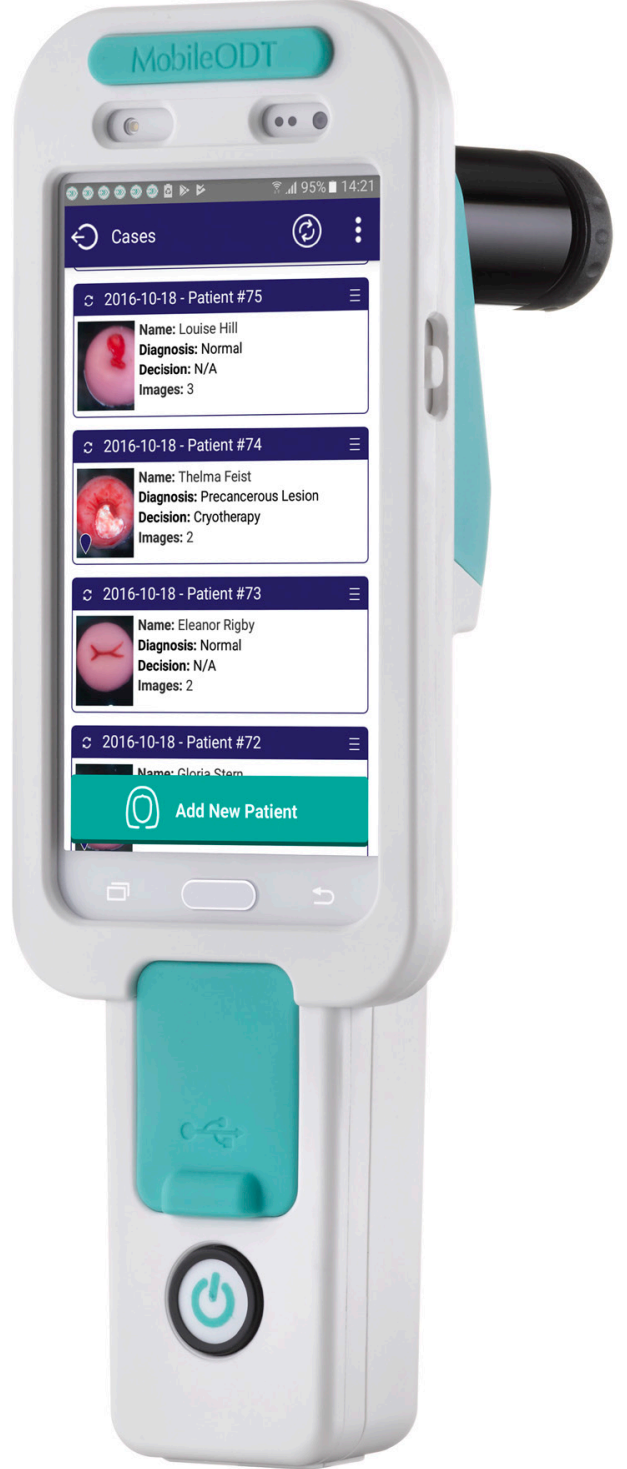

Figure 1 Enhanced visual assessment system, MobileODT.

can allow for a large number of women to be screened in a relatively short amount of time. In a recent metaanalysis, the sensitivities and specificities of patient selfsampling compared with those collected by physicians for detecting HPV were comparable $(74 \%$ and $88 \%$ vs $81 \%$ and $90 \%$, respectively). ${ }^{21}{ }^{22}$ Furthermore, many studies implemented in various international regions show that the majority of women screened by the self-swab method are willing and able to perform the test. ${ }^{23} 24$

The careHPV testing system (QIAGEN, Maryland, USA) is a simple, low-cost, semiportable and robust method for HPV testing, and a new, more economical variant of the traditional Hybrid Capture 2 System that was designed to work in low-resource settings and has been approved by the Chinese FDA (U.S. Food and Drug Administration). ${ }^{19} 25$ The careHPV system can run 90 specimens in approximately 3 hours at the cost of approximately US $\$ 5$ per test. ${ }^{26}$

The aim of this study is to demonstrate that self-swab HPV testing and digital colposcopy can be combined to 
create a rapid, high-volume, cervical cancer screening strategy that can be used in the rural regions of China.

\section{METHODS}

During a 6-day period in July 2018, 3600 Chinese women aged $35-65$ years were screened at three different medical clinics in Kunming Municipality, Xundian County, Yunnan Province. Local health officials recruited potential patients for this study from a list of women registered in their county who had not received prior cervical cancer screening. As such, this represents a non-randomised, convenience sample of study participants. Between 480 and 980 women were screened per day. The majority of the women screened were ethnically Yi, Hui, Dai and Han.

After obtaining written informed consent, the subjects received a brief explanation of HPV and its consequences, as well as instructions on how to obtain a selfsampled vaginal specimen. The specimens were obtained with a vaginal brush designed to work with the careHPV system (careBrush) and then inserted in a tube with the collection medium. Patient-collected samples were then processed on-site by four careHPV machines running simultaneously. All women positive for hrHPV+ results were contacted via cellphone and returned the same day or the following day. Digital colposcopy was then performed with the EVA system after the application of acetic acid for at least $90 \mathrm{~s}$. Colposcopy was performed by one of four physicians. Thin acetowhite lesions were considered positive and suspicious for CIN1 lesions. Thick acetowhite lesions, rapidly appearing lesions, lesions with course mosaicism or punctuation, and lesions with sharp borders were considered positive and suspicious for $\geq$ CIN2 lesions. ${ }^{27}$ If no acetowhite changes were visualised the patients were counselled of the findings and instructed to seek follow-up with the appropriate provider in 1year if possible. The EVA system was then used to obtain digital images (1-3 images per patient). If digital colposcopy was positive for cervical abnormalities, the patients underwent cervical biopsy and then they were triaged to one of two possibilities: (1) Cryotherapy was performed the same day when findings were suspicious for CIN1 lesions. (2) A loop electrosurgical excision procedure (LEEP) was performed the same day when findings were suspicious for $\geq \mathrm{CIN} 2$. If the transformation zone was not fully visible on digital colposcopy, endocervical curettage (ECC) was performed and patients were subsequently contacted by local health authorities for appropriate treatment.

Throughout the screening and treatment process, local doctors and nurses were taught colposcopy techniques and received training to evaluate digital colposcopy images. Additionally, all patients were given their results of the HPV tests and colposcopy. Patients were counselled regarding HPV prevention and transmission. Patients with suspected low-grade lesions (CIN1) (who were treated with cryotherapy) were counselled to have repeat HPV testing in 1year, and those with high-grade lesions
( $\geq$ CIN2) were later contacted with the results of their LEEP and were counselled to follow-up at the regional hospital for appropriate management.

\section{Patient and public involvement}

Immediately after self-swabbing, 600 women were interviewed regarding their understanding of the instructions and their comfort level obtaining the self-swabs. These data will be reported in a separate publication.

\section{RESULTS}

A total of 3600 women, aged 35-65 years, were screened by four physicians in 6 days. The mean age of all women screened was 50.2 years old. Two hundred and sixteen of the 3600 women were hrHPV+ $(6.0 \%)$. Of these 216 women, $168(77.8 \%)$ underwent digital colposcopy. Of the remaining $48 \mathrm{hrHPV}+$ women (22.2\%), 36 chose to follow up at a regional hospital because they were unable to immediately return for colposcopy within 24 hours and 12 refused further evaluation. Of the women who underwent digital colposcopy, 23 were positive for suspected CIN1 (13.7\%) and 17 were positive for suspected $\geq$ CIN2 $(10.1 \%)$. In 26 patients digital colposcopy could not adequately evaluate the entire transformation zone $(15.4 \%)$ and ECC was performed in these patients. One patient with advanced cervical cancer was sent to a referral medical centre and one woman chose to have LEEP performed at a regional medical centre. Twenty of the 23 biopsies performed on the women suspected of having CIN1 confirmed this diagnosis (87\%), whereas one showed CIN2 (4.3\%) and two were negative for dysplasia $(8.7 \%)$. Of the 16 women who were suspected to have $\geq$ CIN2 lesions on digital colposcopy, $\geq$ CIN2 pathology was confirmed in $15(93.8 \%)$, with 10 CIN2 $(62.5 \%)$ and 5 CIN3 (31.3\%) (figure 2). ECC identified three additional women with CIN2 who were contacted for further treatment (figure 3).

\section{DISCUSSION}

As the success of cervical cancer screening programmes have dramatically decreased the rates of cervical cancer in developed nations, the global burden of this disease falls in areas of limited resources. It is estimated that the number of women aged 35-64years in rural China exceeds 150 million. ${ }^{28}$ As the vast majority of these women has never been screened, modalities must be developed that allow for the screening and treating of large numbers of women. We propose a screen-and-treat model that is low-cost, rapid and capable of being implemented on a large scale, as evidenced by the ability to screen 3600 women in less than 1 week. We have previously described a similar strategy for cervical cancer screening among Cambodian women. ${ }^{19}$

The first component of this model consists of selfobtained HPV specimens for rapid testing. Prior studies have demonstrated that self-sampling has a higher 


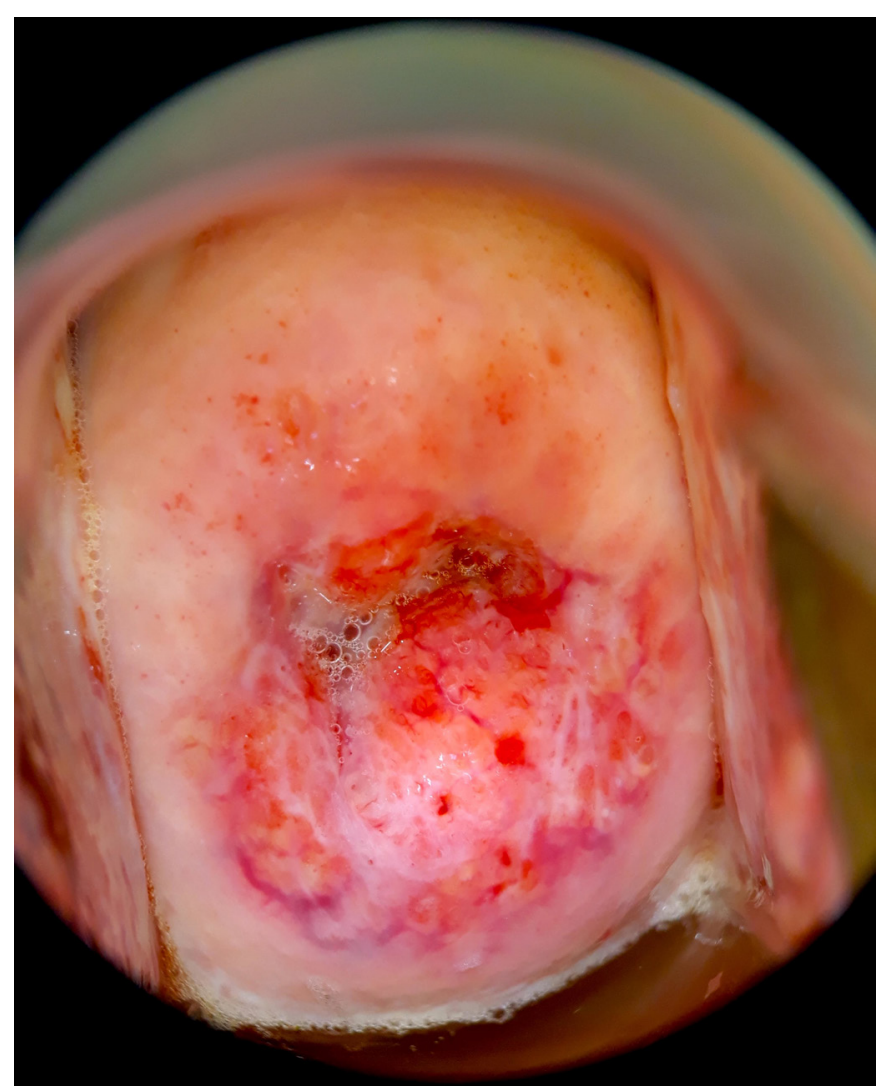

Figure 2 Cervical intraepithelial neoplasia 3 (CIN3).

sensitivity than Pap smears in detecting $\geq$ CIN2 lesions and are only marginally lower in sensitivity than physicianobtained cervical specimens. ${ }^{21} 22$ It would be practical for women in China to obtain self-collected HPV specimens, which would then undergo testing. Rapid, same-day testing is useful because it can decrease loss to follow-up which can be highly problematic because of geographical and transportation issues in rural regions of China. The problem of loss to follow-up was illustrated even in our same-day screen-and-treat study as 36 of 216 (16.67\%) hrHPV+ women were unable to return for same-day colposcopy and 16 of the 36 women never followed up at their regional medical centre.

The highly portable careHPV system is capable of running 90 samples in approximately 3 hours, at a cost of approximately US $\$ 5$ per test. A newer PCR-based HPV testing system has been developed in the 2 years since our screening campaign was performed (Ampfire, Atila Biosystems, California, USA). This new, highly portable, PCR-based system is even faster than careHPV, taking only 1 hour to run the equivalent number of specimens, is less labour-intensive, does not require storage of specimens in liquid media, does not require refrigeration of the swabs and is able to identify specific hrHPV genotypes. ${ }^{29}$ Additionally, PCR-based systems are significantly more sensitive for detecting hrHPV+ on self-swab specimens than older hybrid capture (signal amplification) based systems. ${ }^{21} 2230$ Regardless of the type of system used, rapid testing is a critical aspect of this model as it allows for same-day treatment.

The second component of this proposed model is to perform digital colposcopy on all hrHPV+ women with a highly portable system, such as the EVA system used in this study. Given the huge number of women in China who have never been screened as compared with the number of physicians available, digital colposcopy can be performed by mid-level providers such as nurses or midwives who have had adequate training. Digital images obtained have excellent resolution and areas of question can be magnified for better interpretation (figure 2). In addition, captured images can be used for continued education of mid-level providers, and the images can be sent electronically, through a secure, cloud-based portal, to an expert colposcopist for consultation of difficult cases.

Previously, Newman et al described a cervical cancer screening strategy that combined HPV testing with digital colposcopy using the Gynocular system (Gynius AB, Sweden). ${ }^{31}$ Their study, also performed in the Yunnan Province, was different in that physicians obtained the HPV swabs and then immediately performed digital colposcopy. Their HPV swabs were sent out to a regional medical centre. Additionally, they did not follow a seeand-treat approach and based their treatment on biopsies obtained during the colposcopy.

The third component of this screen-and-treat model is to use the clinical impression obtained from the colposcopic images to determine immediate and appropriate treatment. As shown in this study, the images obtained with digital colposcopy are able to accurately distinguish between CIN1 and $\geq$ CIN2 lesions. Of the $16 \geq$ CIN2 pathologically confirmed lesions, 15 were identified as $\geq$ CIN2 on digital colposcopy. Women with suspected CIN1 lesions could be treated with cryotherapy or thermocoagulation and those with suspected $\geq$ CIN2 lesions could undergo a LEEP procedure. While biopsy-proven CIN1 lesions do not need to be treated, in screen-andtreat programmes such as these where the diagnosis is not confirmed with pathology, the WHO recommends that these women undergo an ablative procedure. ${ }^{32}$

The current WHO guidelines for low-resource settings, such as rural China, conditionally recommend using a strategy of screening with HPV testing followed by visualization with acetic acid (VIA), over screening with an HPV test followed by colposcopy. The guidelines further recognise that this conditional recommendation is based on 'very low quality of evidence' ${ }^{32}$ WHO guidelines justify this by stating that 'there may be more resource implications with colposcopy due to increased training of providers, quality control, waiting time, and the potential for more women to be lost to follow-up. ${ }^{32}$ These recommendations were made prior to the advent of highquality, portable, digital colposcopes such as the system used in this study, which does not carry these limitations. Moreover, WHO guidelines acknowledge that there may be fewer $\geq$ CIN2 recurrences with the HPV test followed 


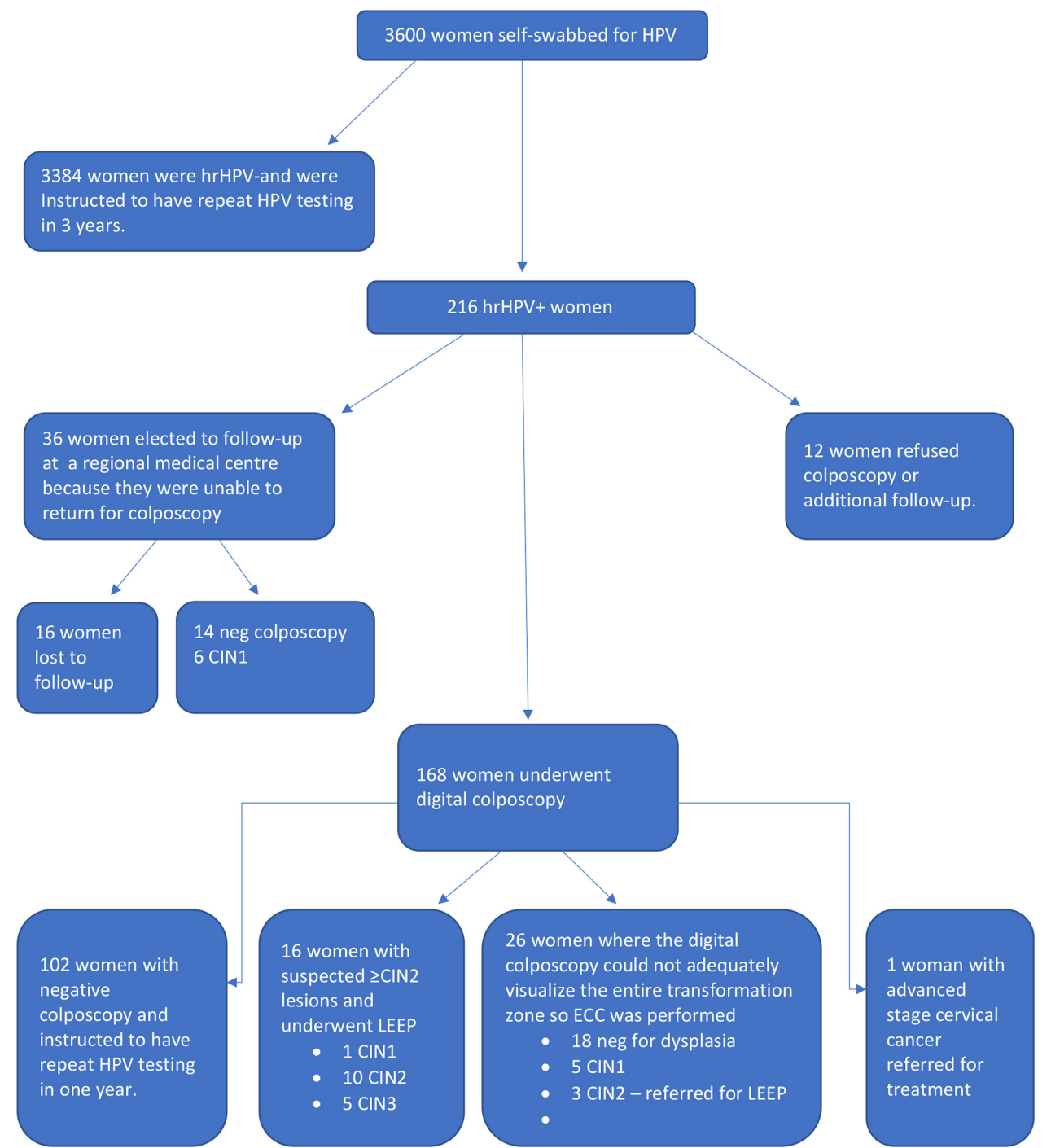

Figure 3 Study results flow chart. CIN, cervical intraepithelial neoplasia; ECC, endocervical curettage; HPV, human papillomavirus; hrHPV, high-risk HPV; LEEP, loop electrosurgical excision procedure.

by colposcopy without biopsy, as well as fewer cervical cancers, and fewer deaths than with an HPV test followed by VIA. As such, this study, and previously performed studies, suggest that WHO guidelines could be modified in countries with the necessary resources to favour digital colposcopy over VIA after HPV testing. ${ }^{19}$

Our proposed screening strategy also slightly differs from current WHO recommendations of using cryotherapy as the primary treatment modality in all screen-and-treat protocols that do not have diagnostic histology. ${ }^{32}$ The WHO recommends that for screen-andtreat programmes LEEP should only be used in lesions that occupy $>75 \%$ of the ectocervix or when the lesion extends into the cervical os. Additionally, the WHO also currently recommends either LEEP or cryotherapy in women with histologically confirmed $\geq$ CIN2 but acknowledges that the overall benefits may be greater with LEEP because it is probable there is less recurrence of dysplasia with LEEP as compared with cryotherapy. ${ }^{33}$ We propose that in screening campaigns that are able to use highquality digital colposcopy, LEEP can be performed in colposcopically suspected $\geq$ CIN2 (without waiting for histopathological confirmation) as this study and a prior published study using EVA demonstrated that colposcopic impressions were accurately able to distinguish between CIN1 and $\geq \mathrm{CIN} 2 .{ }^{19}$

\section{Strengths and limitations}

Overall, the main strength of our study is that it demonstrated the ability to screen and treat 3600 women in a less than a week with only four skilled providers. Additionally, it demonstrated the usefulness of handheld digital colposcopy in differentiating between CIN1 and $\geq$ CIN2 lesions.

The main limitation of our study was the observed prevalence of hrHPV infection of only $6.0 \%$. This rate is somewhat lower than those previously observed for eastern areas in Yunnan Province, which demonstrate a range from $8.3 \%$ to $11.6 \%$, depending on both location and ethnicity of the study subjects. ${ }^{34} 35$ There are several possible explanations for this finding. First, the careHPV system uses signal amplification technology that is less sensitive than PCR-based systems that have 
been used to determine the aforementioned prevalence rates. ${ }^{2130}$ Second, as mentioned previously, self-swab specimens of the vagina have been shown to be less sensitive than provider-obtained specimens. A study by Belinson $e t$ al of more 2600 women in Shanxi Province, China that used the careHPV system demonstrated that self-swabs were able to detect $80.9 \%$ of $\geq \mathrm{CIN} 2$ lesions whereas provider-obtained endocervical swabs detected $97.9 \%{ }^{36}$ Third, the mean age of patients screened in this study (50.2 years) was significantly higher than in other HPV prevalence studies. Previous studies have shown that the percentage of hrHPV+ women is lower in this age range than in younger women. ${ }^{37}$ Additionally, due to menopausal changes, the transformation zone could not be sufficiently visualised in a large percentage of the colposcopies performed during this study. Fourth, the majority of women in this study were of Yi, Hui and Dai ethnicities. The Yi, Hui and Dai are generally considered socially conservative (with potentially less sexual partners) and this may contribute to a lower rate of HPV infection in this population. Previous studies of Dai women in Yunnan Province have shown a hrHPV+ rate of only $7.1 \%$ as determined by PCR-based testing. ${ }^{13}$ It is likely, therefore, that a combination of these factors contributed to our low prevalence rate.

Another limitation of this study is that it was not designed as a cost-effectiveness study. While we know the costs of supplies, HPV testing, specimen processing, and the transportation and housing of the study personnel, there were significant donations in kind (labour of the study personnel, support of local Chinese healthcare workers and facility fees) that would need to be added to calculate the true costs of this screening study. However, the costs of implementing this screening strategy in China would be significantly less per patient than our per patient costs as there would be significant economies of scale that could be implemented to lower the per patient cost.

\section{CONCLUSION}

Cost-effective, efficient and large-scale cervical cancer screening strategies are desperately needed in lowresource settings. These strategies must be implemented to screen millions of women in China, and over a billion women in resource-poor countries, who have never been screened for cervical cancer. Our proposed screen-andtreat model that combines high-volume, rapid self-swab HPV testing followed by digital colposcopy has the potential to achieve this goal in the near future and at a relatively low cost. Future studies should examine the use of newer PCR-based HPV testing systems as they are faster, less labour-intensive, easier to use and may increase sensitivity as compared with hybrid capture based systems.

\section{Author affiliations}

${ }^{1}$ Obstetrics and Gynecology, The George Washington University School of Medicine and Health Sciences, Washington, DC, USA
${ }^{2}$ The Center for Vulvovaginal Disorders, Washington, DC, USA

${ }^{3}$ Yale University, New Haven, Connecticut, USA

${ }^{4}$ Beijing United Family Hospitals and Clinics, Beijing, China

${ }^{5}$ Department of Psychological and Brain Sciences, Johns Hopkins University,

Baltimore, Maryland, USA

${ }^{6}$ MobileODT, Tel Aviv, Israel

${ }^{7}$ Annapolis Dermatology Center, Annapolis, Maryland, USA

${ }^{8}$ Bellingham Bay Family Medicine, Washington, DC, USA

${ }^{9}$ Pacific Northwest Urology Specialists, Washington, DC, USA

${ }^{10}$ Engineering, University of California Berkeley, Berkeley, California, USA

${ }^{11}$ Duke University, Durham, North Carolina, USA

Twitter Andrew Goldstein @andrewgoldstei2

Acknowledgements MobileODT provided technical assistance during the data acquisition phase of this study. Qiagen provided technical assistance during the data acquisition phase of this study.

Contributors AG: Study conception, study design, data acquisition, data analysis drafting work, final approval, agreement to be accountable for the work. LSG: Study conception, study design, data acquisition, revising work, final approval, agreement to be accountable for the work. RL: Study conception, study design, data acquisition, revising work, final approval, agreement to be accountable for the work. SB: Data acquisition, revising work, final approval, agreement to be accountable for the work. JW: Data acquisition, revising work, final approval, agreement to be accountable for the work. SAS: Data acquisition, revising work, final approval, agreement to be accountable for the work. GB: Data acquisition, revising work, final approval, agreement to be accountable for the work. GRG: Study conception, study design, data acquisition, drafting work, final approval, agreement to be accountable for the work. KDO'K: Data acquisition, revising work, final approval, agreement to be accountable for the work. SCO'K: Data acquisition, revising work, final approval, agreement to be accountable for the work. MO'K: Data acquisition, revising work, final approval, agreement to be accountable for the work. TO'K: Data acquisition, revising work, final approval, agreement to be accountable for the work. ARG: Study conception, study design, data acquisition, revising work, final approval, agreement to be accountable for the work. AZ: Study design, data acquisition, revising work, final approval, agreement to be accountable for the work.

Funding This work was supported by the Gynecologic Cancer Research Foundation, the United Foundation for China's Health, and regional health offices of the Peoples Republic of China.

Competing interests AG: President of the Gynecologic Cancer Research Foundation: a non-profit 501(c)3 corporation. Received research funding from the Gynecologic Cancer Research Foundation. RL: Director, United Foundation for China's Health, a non-profit 501(c)3 corporation. Investor, MobileODT. GB: Was an employee of MobileODT during the data acquisition phase of this study. GRG: Member, Board of Directors, the Gynecologic Cancer Research Foundation: a nonprofit 501(c)3 corporation. AZ: employee, United Foundation for China's Health, a non-profit 501(c)3 corporation.

Patient consent for publication Not required.

Ethics approval United Family Hospitals Institutional Review Board Beijing, China (approval number: UFHIRB. E2018-0002).

Provenance and peer review Not commissioned; externally peer reviewed.

Data availability statement Data are available in a public, open access repository. Extra data can be accessed via the Dryad data repository at http://datadryad.org/ with the https://doi.org/10.5061/dryad.8w9ghx3gr.

Open access This is an open access article distributed in accordance with the Creative Commons Attribution Non Commercial (CC BY-NC 4.0) license, which permits others to distribute, remix, adapt, build upon this work non-commercially, and license their derivative works on different terms, provided the original work is properly cited, appropriate credit is given, any changes made indicated, and the use is non-commercial. See: http://creativecommons.org/licenses/by-nc/4.0/.

ORCID iD

Andrew Goldstein http://orcid.org/0000-0003-3486-2402

\section{REFERENCES}

1 World Health Organization. Cancer, key Statics, 2019. Available: https://www.who.int/cancer/resources/keyfacts/en/ 
2 Progress in cervical cancer prevention: the CCA report card, 2015. Available: https://www.iccp-portal.org/resources/progress-cervicalcancer-prevention-cca-report-card-0

3 Mo X, Gai Tobe R, Wang L, et al. Cost-effectiveness analysis of different types of human papillomavirus vaccination combined with a cervical cancer screening program in mainland China. BMC Infect Dis 2017;17:502.

4 Gu XY, Zheng RS, Sun KX, et al. [Incidence and mortality of cervical cancer in China, 2014]. Zhonghua Zhong Liu Za Zhi 2018;40:241-6.

5 Bruni L, Diaz M, Castellsagué X, et al. Cervical human papillomavirus prevalence in 5 continents: meta-analysis of 1 million women with normal cytological findings. J Infect Dis 2010;202:1789-99.

6 Forman D, de Martel C, Lacey CJ, et al. Global burden of human papillomavirus and related diseases. Vaccine 2012;30:F12-23.

7 Huang S, Afonina I, Miller BA, et al. Human papillomavirus types 52 and 58 are prevalent in cervical cancers from Chinese women. Int $J$ Cancer 1997;70:408-11.

8 Lo KWK, Wong YF, Chan MKM, et al. Prevalence of human papillomavirus in cervical cancer: a multicenter study in China. Int $J$ Cancer 2002;100:327-31.

9 Liu J, Rose B, Huang X, et al. Comparative analysis of characteristics of women with cervical cancer in high-versus low-incidence regions. Gynecol Oncol 2004;94:803-10.

$10 \mathrm{He}$ J, Chen WQ. Chinese cancer registry annual report. Beijing, China. Beijing, People's Republic of China: Military Medical Science Press, 2012.

11 Yunnan Provincial Census Office. The 2000 census of Yunnan Province, 1st EDN. Kunming, China: Yunnan Science and Technology Publishing House, 2002.

12 Yunnan poverty maps, 2019. Available: https://sedac.ciesin. columbia.edu/data/set/povmap-small-area-estimates-povertyinequality/maps/2

13 Baloch Z, Yasmeen N, Li Y, et al. Prevalence and risk factors for human papillomavirus infection among Chinese ethnic women in southern of Yunnan, China. Braz J Infect Dis 2017;21:325-32.

14 Bradford L, Goodman A. Cervical cancer screening and prevention in low-resource settings. Clin Obstet Gynecol 2013;56:76-87.

15 WHO Guidelines Approved by the Guidelines Review Committee. Who guidelines: use of cryotherapy for cervical intraepithelial neoplasia. Geneva: World Health Organization, 2011.

16 Manga S, Parham G, Benjamin N, et al. Cervical cancer screening in Cameroon: interobserver agreement on the interpretation of digital cervicography results. J Low Genit Tract Dis 2015;19:288-94.

17 Bateman AC, Parham GP, Sahasrabuddhe VV, et al. Clinical performance of digital cervicography and cytology for cervical cancer screening in HIV-infected women in Lusaka, Zambia. J Acquir Immune Defic Syndr 2014;67:212-5.

18 Liu AH, Gold MA, Schiffman M, et al. Comparison of colposcopic impression based on live colposcopy and evaluation of static digital images. J Low Genit Tract Dis 2016;20:154-61.

19 Thay S, Goldstein A, Goldstein LS, et al. Prospective cohort study examining cervical cancer screening methods in HIV-positive and HIV-negative Cambodian women: a comparison of human papilloma virus testing, visualization with acetic acid and digital colposcopy. BMJ Open 2019;9:e026887

20 Koliopoulos G, Nyaga VN, Santesso N, et al. Cytology versus HPV testing for cervical cancer screening in the general population. Cochrane Database Syst Rev 2017;8:Cd008587.

21 Arbyn M, Smith SB, Temin S, et al. Collaboration on self-sampling and HPV testing. detecting cervical precancer and reaching under screened women by using HPV testing on self-samples: updated meta-analyses. BMJ 2018;363:k4823.

22 Arbyn M, Verdoodt F, Snijders PJF, et al. Accuracy of human papillomavirus testing on self-collected versus clinician-collected samples: a meta-analysis. Lancet Oncol 2014;15:172-83.

23 Oketch SY, Kwena Z, Choi Y, et al. Perspectives of women participating in a cervical cancer screening campaign with community-based HPV self-sampling in rural Western Kenya: a qualitative study. BMC Womens Health 2019;19:75.

24 Morgan K, Azzani M, Khaing SL, et al. Acceptability of women selfsampling versus clinician-collected samples for HPV DNA testing: a systematic review. J Low Genit Tract Dis 2019;23:193-9.

25 Tuerxun G, Yukesaier A, Lu L, et al. Evaluation of careHPV, cervista human papillomavirus, and hybrid capture 2 methods in diagnosing cervical intraepithelial neoplasia grade $2+$ in Xinjiang Uyghur women. Oncologist 2016;21:825-31.

26 Kang L-N, Jeronimo J, Qiao Y-L, et al. Optimal positive cutoff points for careHPV testing of clinician- and self-collected specimens in primary cervical cancer screening: an analysis from rural China. $J$ Clin Microbiol 2014;52:1954-61.

27 Khan MJ, Werner CL, Darragh TM, et al. ASCCP colposcopy standards: role of colposcopy, benefits, potential harms, and terminology for colposcopic practice. J Low Genit Tract Dis 2017;21:223-9.

28 Ministry of Health. China's health statistics yearbook. Beijing: Peking Union Medical College Press, 2013.

29 Kleeman M, Chen X, Reuter C, et al. Evaluation of a novel isothermal amplification assay for rapid HPV detection and genotyping. Poster \#00489, EUROGIN 2018 - International Multidisciplinary HPV Congress, Lisbon, Portugal, 2018.

30 Salazar KL, Duhon DJ, Olsen R, et al. A review of the FDA-approved molecular testing platforms for human papillomavirus. J Am Soc Cytopathol 2019;8:284-92.

31 Newman $\mathrm{H}, \mathrm{Hu} \mathrm{J}$, Li X, et al. Evaluation of portable colposcopy and human papillomavirus testing for screening of cervical cancer in rural China. Int J Gynecol Cancer 2019;29:23-7.

32 WHO Guidelines Approved by the Guidelines Review Committee. WHO guidelines for screening and treatment of precancerous lesions for cervical cancer prevention. Geneva: World Health Organization, 2013.

33 WHO Guidelines Approved by the Guidelines Review Committee. WHO guidelines for treatment of cervical intraepithelial neoplasia 2-3 and adenocarcinoma in situ: cryotherapy, large loop excision of the transformation zone, and cold knife conization. Geneva: World Health Organization, 2014.

34 Sun L-L, Cheng X-M, Li H, et al. Prevalence and risk factors for cervical cytology abnormality in general female population in Qujing of Yunnan Province, China. Chin Med J 2013;126:1989-91.

35 Baloch Z, Li Y, Yuan T, et al. Epidemiologic characterization of human papillomavirus (HPV) infection in various regions of Yunnan Province of China. BMC Infect Dis 2016;16:228.

36 Belinson JL, Hu S, Niyazi M, et al. Prevalence of type-specific human papillomavirus in endocervical, upper and lower vaginal, perineal and vaginal self-collected specimens: implications for vaginal selfcollection. Int J Cancer 2010;127:1151-7.

37 Li Z, Liu F, Cheng S, et al. Prevalence of HPV infection among 28,457 Chinese women in Yunnan Province, Southwest China. Sci Rep 2016;6:21039. 